\title{
Improvement of Transient Stability of Power System Using Solid State Circuit Breaker
}

\author{
${ }^{1}$ Kalai Murugan Amirthalingam and ${ }^{2}$ Raja Prabu Ramachandran \\ ${ }^{1}$ Department of Electrical and Electronics Engineering, Sathyabama University, Chennai, Tamil Nadu, India \\ ${ }^{2}$ Department of Electrical and Electronics Engineering, B.S. Abdur Rahman University, Chennai, Tamil Nadu, India
}

Received 2012-09-21, Revised 2012-10-09; Accepted 2013-06-07

\begin{abstract}
The aim of this study is to use a solid state circuit breaker for the improvement of transient stability margin. Long distance AC transmission is often subject to stability problems, which limits the transmission capability. Interconnected power systems suffer from weakly damped swings between synchronous generator and sub systems. The development of modern power system has lead to an increasing complexity in the study of power system and also presents new challenges to power system stability and in particular to the aspects of transient stability and small signal stability. Transient stability control plays a significant role in ensuring the stable operation of power systems in the event of large disturbances and faults and is thus a significant area of research. This study investigates the improvement of transient stability of a two area system, using Solid State Circuit Breaker which is capable of fast switching in case of faults and thus controlling the real and reactive power flows in a faulted transmission line. Simulations are carried out in Matlab/Simulink environment for two area system model with Solid State Circuit Breaker to analyze the effects of SSCB on transient stability performance of the system. From the simulation results, swing curves of the three phase faulted power system without SSCB gets increases monotonically and thus the system can be considered as unstable whereas the swing curves of the system with an SSCB reaches to a steady state.
\end{abstract}

Keywords: Solid State Circuit Breakers (SSCBs), Simulation, Semiconductors, Transient Stability

\section{INTRODUCTION}

Transient stability is the ability of the system to return to a normal operating state and generators remaining in synchronism, following a large disturbance, such as multiphase short circuit or switching of lines. Modern power system is a complex network comprising of numerous generators, transmission lines, variety of loads and transformers. As a consequence of increasing power demand, some transmission lines are more loaded than was planned when they were built. With the increased loading of long transmission lines, the problem of transient stability after a major fault can become a transmission limiting factor. Now power engineers are much more concerned about transient stability problem due to the blackout in northeast United States, Scandinavia, England and Italy. Power systems exhibit various modes of oscillation due to interaction among the system components. Most of the oscillations are due to synchronous generator rotors swinging relative to each other (Karady, 2002). Stressed power systems are known to exhibit non linear behavior. If the oscillation is not controlled properly, it may lead to a total or partial system outage. If no adequate damping is possible, the oscillations may be sustained for minutes affecting power flows and grow to cause loss of synchronism between systems. Application of power system stabilizer has been one of the first measures to enhance the

Corresponding Author: Kalai Murugan Amirthalingam, Department of Electrical and Electronics Engineering, Sathyabama University, Chennai, Tamil Nadu, India 
damping of power swings. With increasing transmission line loading over long distances, the use of conventional power system stabilizer might in some cases, not provide sufficient damping for power swings. Load changes or faults are the main causes of power oscillations. Following a sudden disturbance on a power system rotor speeds, rotor angular differences and power transfer undergo fast changes whose magnitude are depend upon the severity of disturbance. For a large disturbance, changes in angular differences may be large as to cause the machines to fall out of step which leads to transient instability. There is a large range of disturbances which may occur on a power system, but a fault on a heavily loaded line which requires opening the line to clear the fault is usually of greatest concern. The tripping of a loaded generator or the abrupt dropping of a large load may also cause instability. The effect of short circuits (faults), the most severe type of disturbance to which a power system is subjected, must be determined in nearly all stability studies. During a fault, electrical power from nearby generators is reduced drastically, while power from remote generators is scarcely affected. In some cases, the system may be stable even with a sustained fault, whereas the other systems will be stable only if the fault is cleared with sufficient rapidity (Haque et al., 2004). Whether the system is stable on the occurrence of fault depend not only on the system itself, but also on the type of fault, location of fault, rapidity of clearing and the method of clearing, i.e., whether cleared by sequential opening of two or more breakers or by simultaneous opening and whether or not the faulted line is reclosed. The circuit breakers near the fault disconnect the unhealthy part of the system so that the power transfer can be partially restored, improving the chances of the system remaining stable. The shorter the time to breaker operating, called clearing time, the higher the probability of the system being stable. Solid State Circuit Breakers (SSCB), based on modern high power semiconductors, offer enormous advantages when compared to mechanical circuit breakers with respect to speed and life (Klingbeil et al., 2001). The distortions caused by a three phase short circuit can be limited to a few $100 \mu$ s. In contrast to this, present circuit breaker technology requires at least $100 \mathrm{~ms}$ to clear a fault (Ekstrom et al., 2001). This concept may be used to improve the transient the stability of the power system when fault occurs. The above literature does not deal with the improvement of transient stability using Solid State
Circuit Breaker. This study proposes solid state circuit breaker for the improvement of transient stability.

\section{MATERIALS AND METHODS}

\subsection{Mathematical Model of Multi-Machine Power System}

The dynamics of a single synchronous machine connected to infinite bus bars shown in Fig. 1, is governed by the nonlinear differential Equation 1-3:

$\mathrm{M} \frac{\mathrm{d}^{2} \delta}{\mathrm{dt}^{2}}=\mathrm{P}_{\mathrm{m}}-\mathrm{P}_{\mathrm{e}}$

It is also known that:

$\frac{\mathrm{d} \delta}{\mathrm{dt}}=\omega$

$M \frac{d \omega}{d t}=P_{m}-P_{e}$

A time domain simulation program can solve these equations through step-by-step integration by producing time response of all state variables.

Generator stability is determined by observing the swing curves generated through numerical integration of the system dynamic equations. Figure 2 and $\mathbf{3}$ shows the transient stability phenomenon.

\subsection{Solid State Circuit Breaker 2.2.1. Basics}

The integration of sensible loads, e.g., computers, embedded controllers in desktop as well as in industrial applications results in an increased importance of power quality in electrical grids. Short time interruptions, e.g., due to lightning or short circuit in neighboring circuits, have little influence on the statistic and are simply not taken into account. In medium voltage grids the handling of short circuits is an important issue to increase the power quality. Especially, when considering the increasing short circuit power due to enlarged distributed energy generation in medium voltage systems. Power quality is concerned with such effects as availability, voltage distortion and deviations from normal values (voltage, frequency) over short period of time.

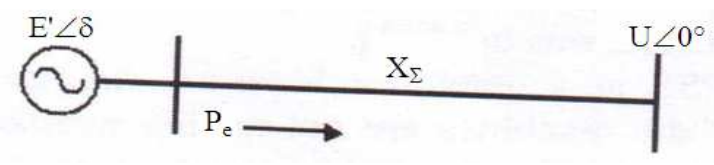

Fig. 1. Single machine to infinite bus 


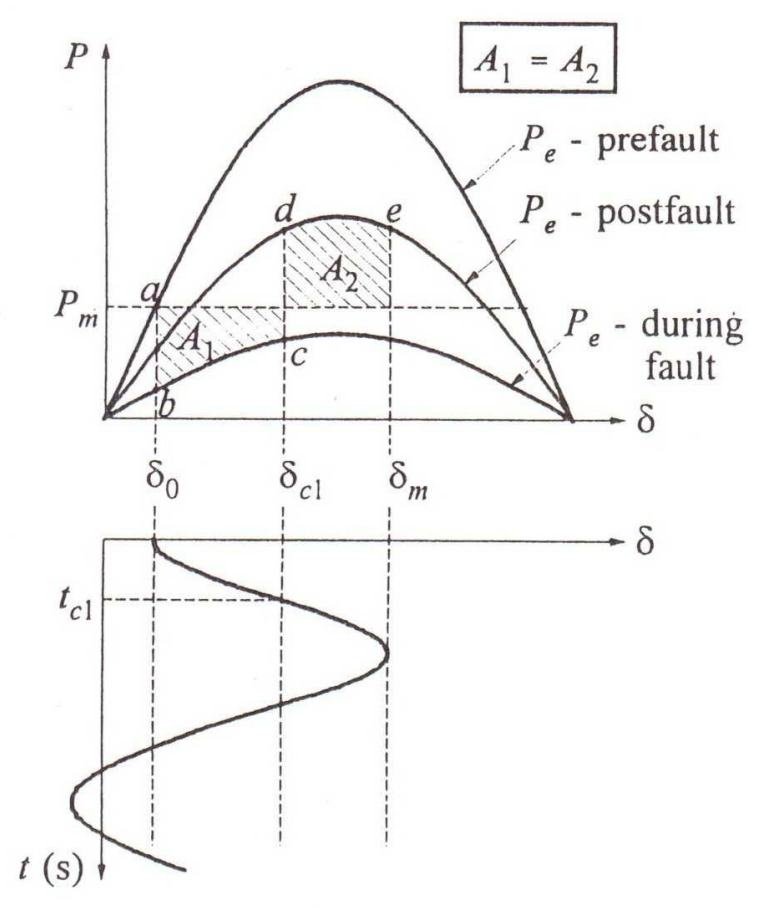

Fig. 2. Response to a fault cleared in tc1 seconds - stable

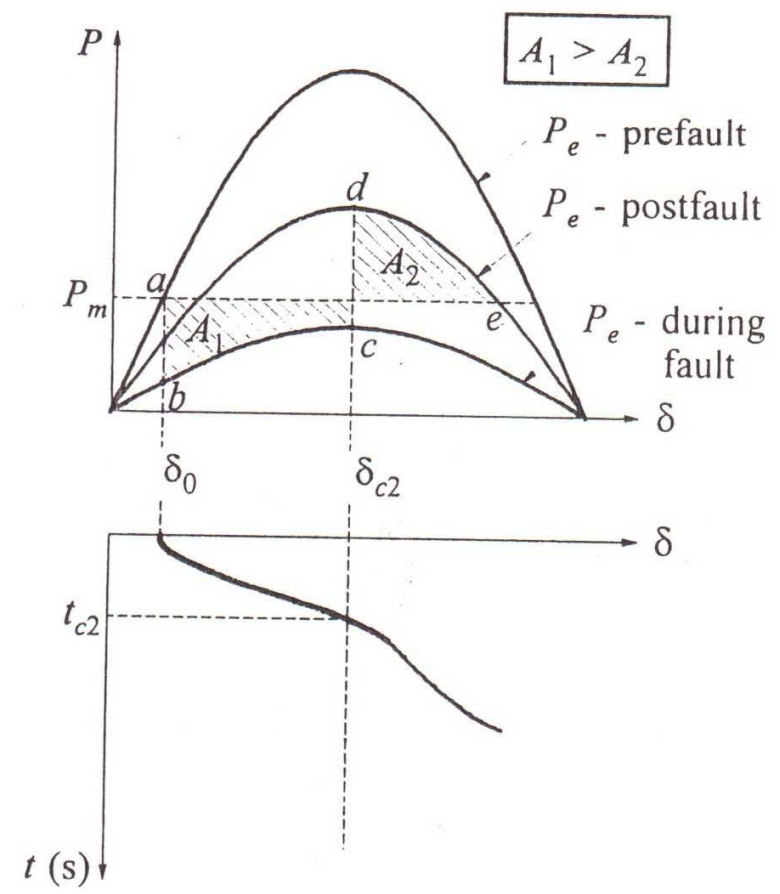

Fig. 3. Response to a fault cleared in tc2 seconds-Unstable
The handling of short circuits in the grid is of great importance in order to provide safety and to achieve high availability on the one hand and high power-quality on the other. The present solutions dealing with short circuit protection are mechanical circuit breakers. After having detected a short circuit or overload situation, some time elapses prior to open the switches mechanically. Subsequently, an arc occurs, which initially has little impact on the current. The current can only quenched at its natural zero crossing that the plasma is significantly cooled down to avoid resignation. As a result, turning off a short circuit will take at least $100 \mathrm{~ms}$ (without detection time). Handling short circuits becomes problematic when considering the increased short circuit power resulting from distributed energy generation in medium voltage systems. As a consequence, it is likely that in the near future classical mechanical circuit breakers will not be able to handle to these currents (Meyer et al., 2004).

In the mechanical circuit breaker, peak current cannot be influenced. Therefore, all network components have to withstand the peak current during the switching period. This current reaches peak values, which are typically 20 times higher than the maximum operating current. This current is below the transient peak current value but can still reach values up to ten times the nominal value. During the short-circuit time, the voltage on the complete medium-voltage grid is significantly reduced. Due to the long turn-off delay of the breaker, sensible loads require UPS support to survive this sag, which is costly and might not be feasible for a complete factory plant (Kunde et al., 2003).

It has already proven that the usage of power semiconductor devices could lead to reduced current values and voltage distortions during short circuit failure. In addition to limiting the fault current, the SSCB can also limit the inrush current (soft start capability), even for capacitive loads, by gradually phasing in the switching device rather than making an abrupt transition from an open to closed position. A SSCB can offer the following advantages: Limited fault current, limited inrush current (soft start) and repeated operations with high reliability and without wear-out, reduced switching surges, improved power quality for un faulted lines (Meyer et al., 2003). So, Solid State Circuit Breakers based on the power semi conductors potentially offer enormous advantages when compared to conventional solutions.

\subsection{Fundamental Behavior}

To analyze the fundamental behavior of the proposed Solid State Circuit Breaker, A single phase equivalent 
circuit is shown in Fig. 4, is used. The grid is represented by voltage source and line impedance. In this example, a pure resistive load is shorted by an ideal short circuit with zero resistance. The typical wave form of the current in and voltage across the SSCB are shown in Fig. 5. At approximately $2 \mathrm{~ms}$, a short circuit occurs and the current rises fast. After a small delay time, SSCB opens. The energy stored in the line inductance is dissipated in high energy varistors, which are connected in parallel with the semiconductors. Consequently, the current decreases. When the current reaches zero, the switch has to block the line to neutral voltage of the grid.

The fault current is given by Equation 4:

$$
i_{f}=\frac{V_{m} X}{Z^{2}} e^{t / \tau}+\frac{V_{m}}{Z} \operatorname{Sin}(v t-\varphi)
$$

\subsection{About the Semiconductor Devices}

SSCBs use power semiconductor devices to provide the power switching, replacing the hard contacts in mechanical circuit breakers. Power electronic device enables the production of power management modules that can handle all of the electronic power control and conversion functions required to move power from the generating and storage sources to the ultimate loads. Some ideal requirements of power devices for use in the converters are like adequate blocking voltage, high turn off current and surge current capabilities, adequate isolation voltages withstand, reverse conducting and active switch in one module, low conduction and switching losses, suitable for parallel and series operation, fast switching; tolerance to $\mathrm{dV} / \mathrm{dt}$ and $\mathrm{dI} / \mathrm{dt}$, good thermal performance and ease of production and control (Celli et al., 2000).

These requirements differ in some points to those for power devices for use as circuit breakers. Low conduction losses are far more important than switching losses, because the ability for fast repetitive switching is not necessary in the circuit breaker. The grid voltage is typically much higher than the maximum blocking voltage of present semiconductors although the ratings have increased significantly. Consequently, the several semiconductors have to be connected in series. Since a high reliability is of utmost importance, redundant devices will be integrated (Meyer and Doncker, 2006). Only devices in press-pack housing are useful because they assure that the switch is still operational after a single semiconductor has failed. Today different semiconductors IGBT, GCT, GTO and IGCT can fulfill the requirements.

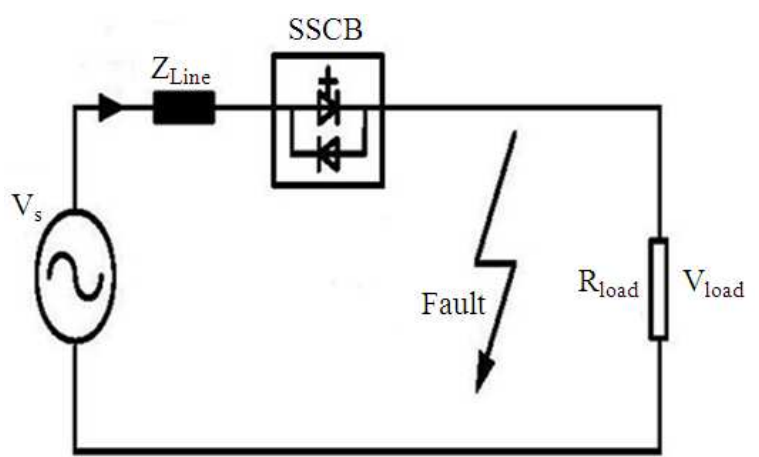

Fig. 4. Single phase equivalent circuit of SSCB

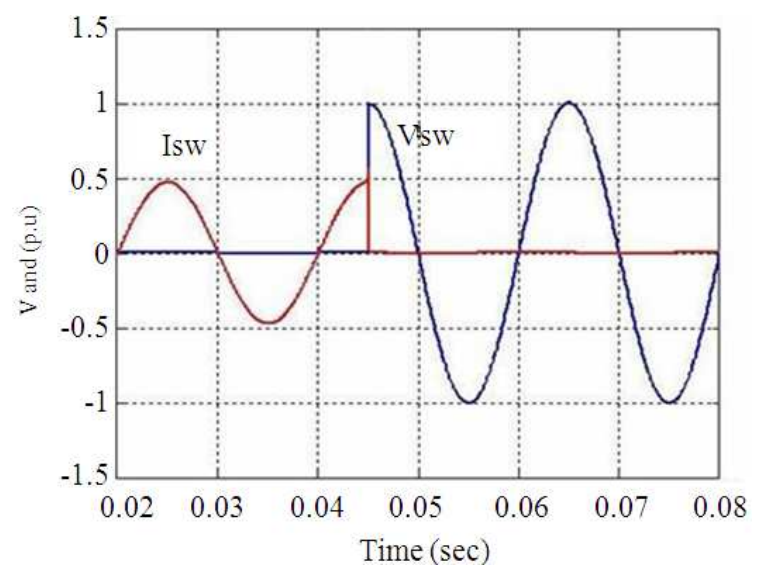

Fig. 5. Voltage and current in ideal SSCB

Power Semiconductors such as Silicon Controlled Rectifiers have been used for SSCBs, but they are only useful for AC circuit breakers. This is because they are latching Thyristor type devices, requiring a zero crossing of the current to turn them off. Other devices such as Power BJTs could be used for SSCBs, this is somewhat impractical because they have low current gain, limited current capability. Newer power MOSFET solves of low current gain and second breakdown, but has limited current capability, unless many devices are connected in parallel.

In contrast to inverter applications, the switching behavior is a minor issue in this application. The conducting behavior and conduction losses are essential. As a result, the IGBT has a disadvantage in circuit breaker application (Steurer et al., 2003). Especially, the on state losses of IGBTs are significantly higher than the losses of a Thyristor based semiconductor (up to 3 times higher per device). However, the IGBT has the advantage that it limits the current internally. Hence the current cannot exceed a certain value. In contrast to this, the current is not limited in a 
Thyristor based semiconductor and the turn off capability is limited. Thus, the deduction time has to be short to assure a safe turn-off. Considering the stray inductance of a medium voltage transformer and the speed of today's deduction technology this time delay can be minimized to not critical values. It becomes obvious that the Thyristor based semiconductors, such as GCT and GTO, are a much better choice for a solid state switch because they have much lower on-state losses. GTOs and GCTs has all of the advantages of other Thyristor type devices like the SCR, but with the added capability of being able to turn off when desired to interrupt current. Since the GTO can be turned off when a high current fault occurs, the device is suitable for use in DC as well as AC SSCBs. Unfortunately, the gate drive current and speed requirements for turning off the GTO are difficult to meet, making the gate drive circuit design very complex (Jian-guo et al., 2010).

It was already shown that the costs associated with losses have a major impact on the overall system and operation costs. Hence, due to the reduced on-state losses, the overall lifecycle costs of the SSCB can be significantly reduced. In addition, a forced air-cooling can be used instead of water-cooling. Consequently from the economical point of view, a thyristor switch could be more competitive when compared with GCT switches. Although a complex auxiliary circuit is needed to enable an active turn-off process, thyristors also offer an economical advantage compared to a GCT solution considering the investment cost, since expensive gate drivers and isolation circuits can be avoided with light triggered SCRs. Of course, the turn-off interval of a thyristor switch is longer compared to a GCT circuit breaker, therefore leading to increased short circuit currents. However, considering the rather large time constants in 50 or $60 \mathrm{~Hz}$ power systems it was found that, the lack of speed ( $1 \mathrm{~ms}$ compared to $400 \mu \mathrm{s}$ ) can be tolerated. Relatively a new semiconductor device called Insulated Gate Commutated Thyristor (IGCT), which is actually an improved version of GTO married to an integrated gate drive circuit, has now been available for more than 10 years. This device has the advantages like high voltage capability, high current gain capability and good electrical ruggedness. The device can be optimized for low on state voltage drop for use in circuit breaker or for low switching losses for use in Power Converters. Overall, these considerations lead to conclusion that forced commutation circuits still can offer an interesting alternative for circuit breaker applications in medium voltage systems.

\section{RESULTS}

Simulink model of a power system with a single machine connected to two loads without SSCB is shown in Fig. 6. The short circuit fault is represented as a low resistance. The power angle curve is shown in Fig. 8. The rotor angle increases as the real power taken by the load decreases and the system may become unstable.

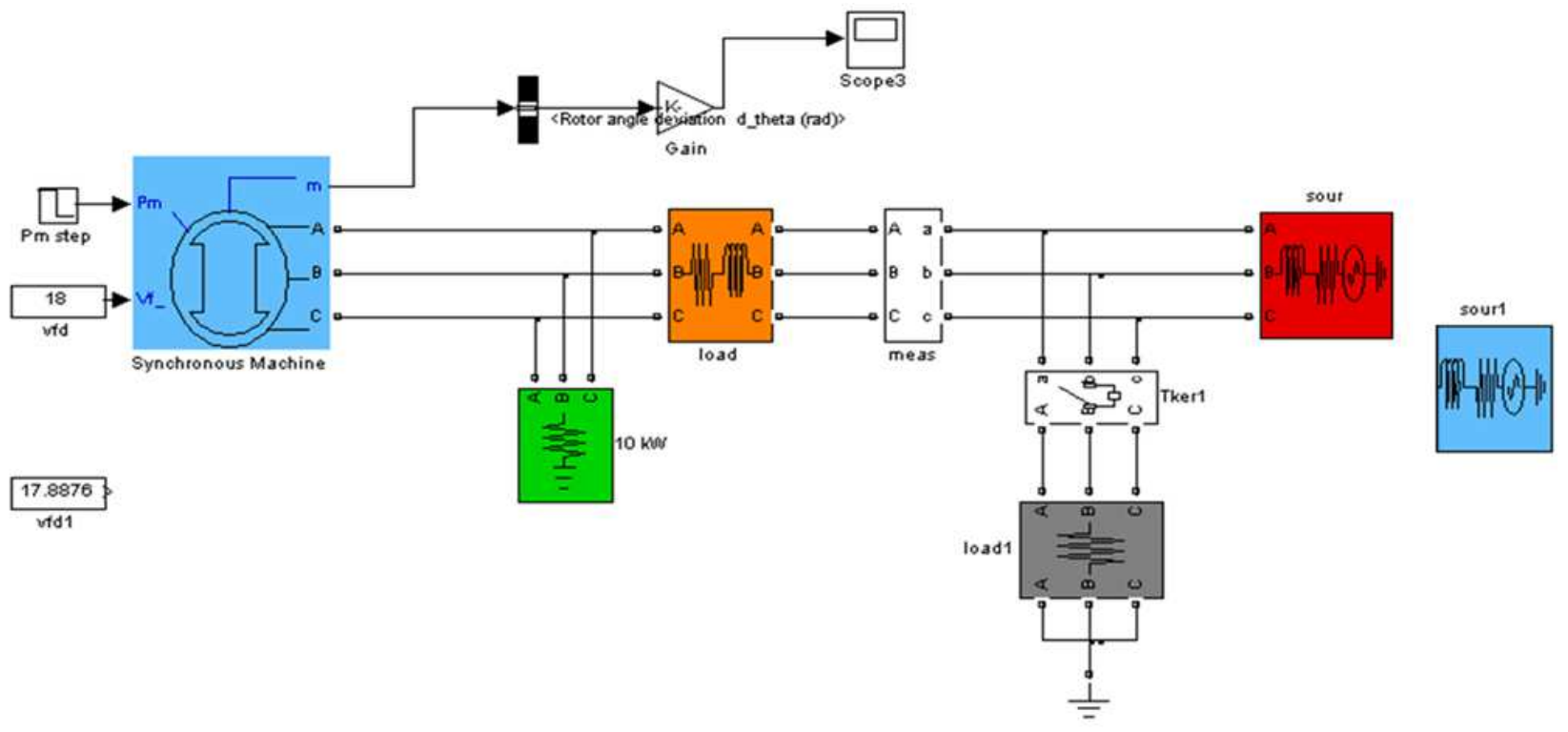

Fig. 6. Power system model without SSCB 


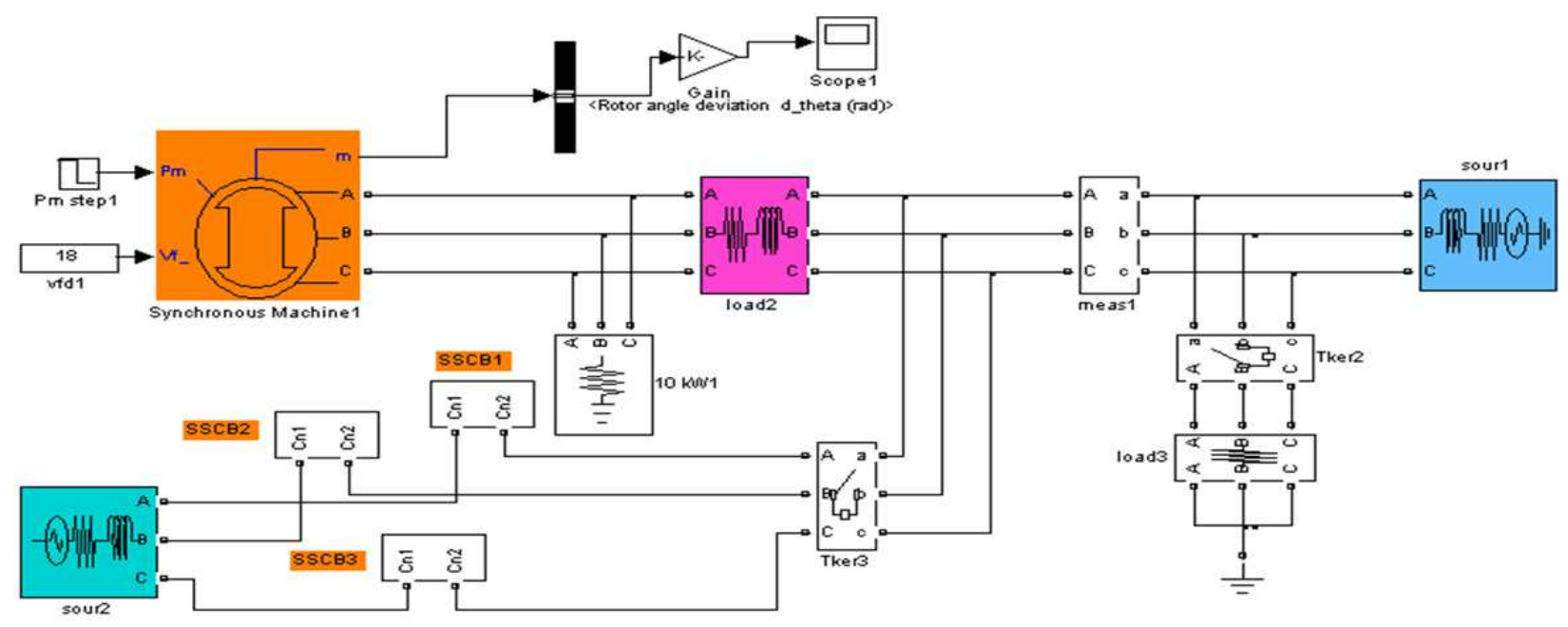

Fig. 7. Power system model with SSCB

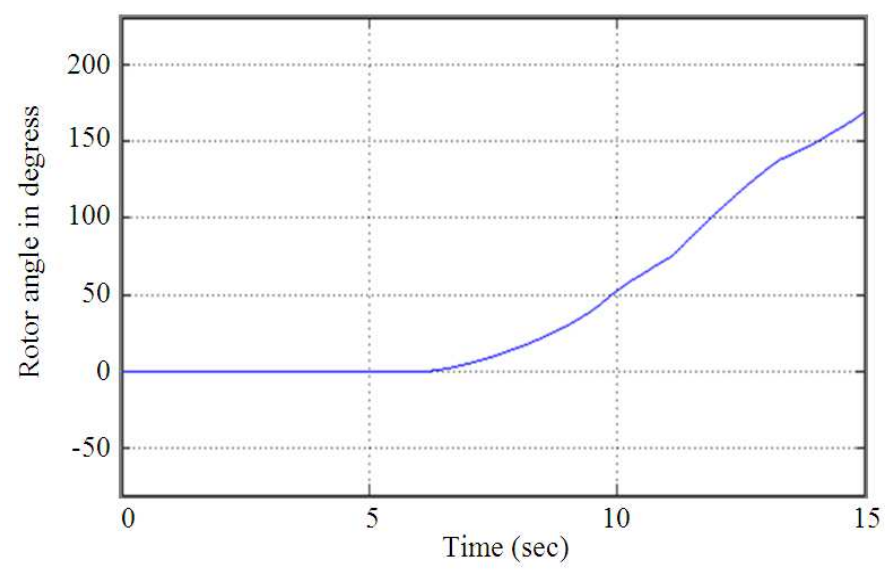

Fig. 8. Power angle curve without SSCB

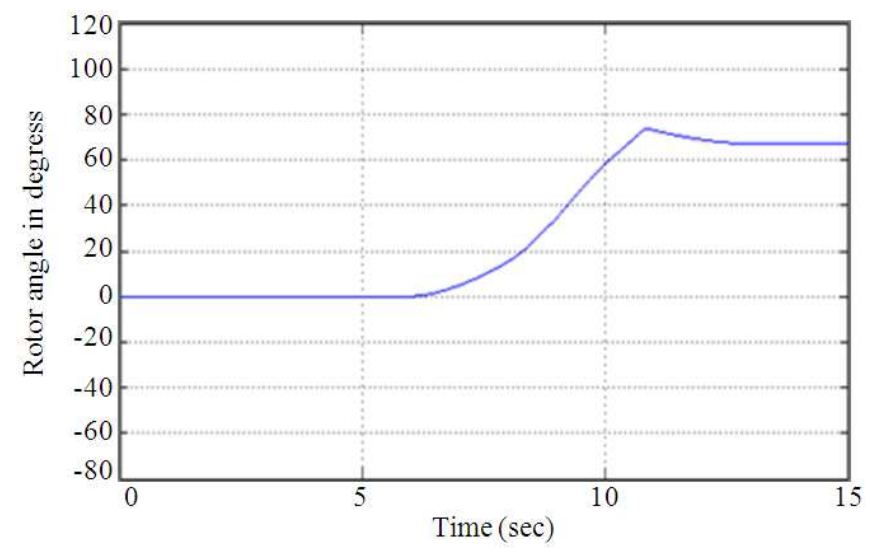

Fig. 9. Power angle curve without SSCB 
The Simulink model of a power system with SSCB is shown in Fig. 7. The SSCB is opened to isolate the faulty section faster than the mechanical circuit breaker. The corresponding rotor angle variation is shown in Fig. 9. It can be observed that the rotor angle reaches the steady state value. Therefore the system is stable.

\section{DISCUSSION}

This study deals with the transient stability improvement of power system using SSCB. Through proper system design, solid state breakers can enable advanced power systems to achieve their full potential for providing loads with high quality power with minimal interruptions. Recently SSCBs offer the possibility of interrupting fault currents very fast hence the SSCB detects the fault current very fast (within a few microsecond), as the fault current rises very fast. So SSCBs also improves the transient stability.

\section{CONCLUSION}

This study investigates the capability of SSCB for the improvement of transient stability of a inter area power system. A power system with an SSCB is modeled and analyzed. From the simulation results it is observed that transient stability of the power system is improved by using SSCB when the fault occurs. When the fault occurs, the SSCB acts faster than the conventional circuit breaker, the power angle settles to a new value and the system becomes stable. These results are in line with the predications. In addition to the advantage of SSCB in improving the transient stability it has other advantages like low manufacturing cost, fast switching and high reliability.

\section{REFERENCES}

Celli, G., F. Pilo, R. Sannais and M. Tosi, 2000. A custom power protection device controlled by a neural network relay. Proceedings of the IEEE Power Engineering Society Summer Meeting, Jul. 16-20, IEEE Xplore Press, Seattle, WA., pp: 13841389. DOI: 10.1109/PESS.2000.868727

Ekstrom, A., P. Bennich, M.D. Oliveira and A. Wilkstrom, 2001. Design and control of a currentcontrolled current limiting device. Proceedings of the Conference European Power Electronics (EPE’01), Graz, Austria.
Haque, M.H., 2004. Improvement of first swing stability limit by utilizing full benefit of shunt FACTS devices. IEEE Trans. Power Syst., 19: 1894-1902. DOI: $10.1109 /$ TPWRS.2004.836243

Jian-guo, M., W. Li and H. Jie, 2010. Research on main circuit topology for a novel dc solid-state circuit breaker. Proceeding of the 5th IEEE Conference on Industrial Electronics and Applications, Jun. 15-17, IEEE Xplore Press, Taichung, pp: 926-930. DOI: 10.1109/ICIEA.2010.5515678

Karady, G.G., 2002. Improving transient stability using generator tripping based on tracking rotor-angle. IEEE Power Eng. Soc. Winter Meet., 2: 1113-1118. DOI: 10.1109/PESW.2002.985183

Kunde, K., M. Kleimaier, L. Klingbeil, H.J. Hermann and C. Neumann et al., 2003. Integration of fast acting Electronic Fault Current Limiters (EFCL) in medium-voltage systems. Proceedings of the 17 th International Conference on Electricity Distribution, May 12-15, Barcelona, pp: 1-9.

Klingbeil, L., W. Kalkner and C. Heinrich, 2001. Fast acting solid-state circuit breaker using state-of-theart power-electronic devices. Proceedings of the European. Conference Power Electron Application (PEA' 2001), Graz, Austria.

Meyer, C., S. Schroder and R.W.D. Doncker, 2004. Solid-state circuit breakers and current limiters for medium-voltage systems having distributed power systems. IEEE Trans. Power Elect., 19: 1333-1340. DOI: 10.1109/TPEL.2004.833454

Meyer, C., S. Schroder and R.W.D. Doncker, 2003. Integration of solid state switches into mediumvoltage grids. Proceedings of the European Conference Power Electron Application, (EPE’03), Toulouse, France.

Meyer, C. and R.W.D. Doncker, 2006. LCC analysis of different resonant circuits and solid-state circuit breakers for medium-voltage grids. IEEE Trans. Power Delivery, 21: 1414-1420. DOI: 10.1109/TPWRD.2005.861334

Steurer, M., K. Fröhlich, W. Holaus and K. Kaltenegger, 2003. A novel hybrid current-limiting circuit breaker for medium voltage: Principle and test results. IEEE Trans. Power Delivery, 18: 460-467. DOI: 10.1109/TPWRD.2003.809614 